\title{
TRANSCATHETER VENUS P VALVE IMPLANTATION AT PULMONARY POSITION- POST TOF REPAIR WITH SEVERE PR- INITIAL CASE IN PAKISTAN
}

\author{
Amjad Mahmood, Khurram Akhtar, Nadeem Sadiq, Shakeel Qureshi, Worakan Promphan, Hajira Akbar
}

Armed Forces Institute of Cardiology (AFIC)/National Institute of Heart Diseases (NIHD), Rawalpindi Pakistan

\begin{abstract}
After total correction for tetrolgy of fallot (TOF), right ventricle behaves in an unpredictable manner depending on type of right ventricular outflow tract (RVOT) reconstruction and surgical expertise of infundibular muscle resection. We are reporting a 23 years old girl who underwent total correction at two years of age. RVOT was reconstructed with native pericardial patch. Gradually she developed breathlessness and occasional chest pain. Echocardiograghy revealed hugely dilated right ventricle (RV) with gross pulmonary regurgitation and RV dysfunction. Cardiac MRI also calculated right ventricular end systolic volume (RVESV) $57 \mathrm{ml} / \mathrm{m}^{2}$ and right ventricular end diatolic volume (RVEDV) $157 \mathrm{ml} / \mathrm{m}^{2}$.

We decided to implant transcatheter venus $p$-valve at pulmonary position. The procedure went successful having competent pulmonary valve and improved RV function. Total fluoro time was 36.4 minutes and total procedural time was two hours. This procedure was done first time in Pakistan with optimal results.
\end{abstract}

Keywords: Right ventricular outflow tract, Branch pulmonary arteries, Pericardial patch, Venus $p$-valve.

This is an Open Access article distributed under the terms of the Creative Commons Attribution License (http://creativecommons.org/licenses/by/4.0), which permits unrestricted use, distribution, and reproduction in any medium, provided the original work is properly cited.

\section{INTRODUCTION}

Transcatheter percutaneous pulmonary valve implantation has become an effective and compliant modality for most of tetralogy correction patients needing pulmonary valve replacement after total correction almost two decades ago $^{1-3}$. Melody and Sapien valves are implanted exclusively when homografts or conduits are used to repair right ventricle outflow tracts (RVOT) for total correction of tetralogy of fallot (TOF). But in many cases repaired earlier transannular patch technique was used instead of homograft which leads large andaneurysmal RVOT which requires larges sized valves as compared to previously available 4 . In this scenario this became mendatory to have larger valve suitable for which can be implanted in native RVOT having transannular patch with significant dilatation.

For this purpose the Venus $p$-valve (Venus Med Tech, Shanghai, China) self-expandable Nitinol multi-level support frame with tri-leaflet porcine pericardial tissue valve (fig-1) designed

Correspondence: Dr Amjad Mahmood, Peads Department, AFIC/NIHD Rawalpindi Pakistan to be implanted in a native patched RVOT. Trials of its use have already been made ${ }^{5,6}$ with promising results still pending $\mathrm{CE}$ certification and FDA approval 7-10. We are sharing our initial experience on the technicality and effectiveness of percutaneous implantation of the venus $p$-valve at the pulmonary position in patients with native RVOT.

\section{CASE REPORT}

A 23 year girl was admitted with chest pain and breathlessness NYHA III along with occasional tachycardia. She underwent TOF repair at the age of two years. We planned transcatheter venus $p$-valve insertion at RVOT as she had severe pulmonary regurgitation leading to gradually failing RV. Echocardiograghy revealed hugely dilated right ventricle (RV) with gross pulmonary regurgitation and RV dysfunction. Tricuspid annular plane systolic excursion (TAPSE) was $10 \mathrm{~mm}$. Cardiac MRI further elaborated RVOT, MPA and brach PA anatomy and helped to asses estimated size of the venus $p$-valve. Cardiac MRI also calculated right ventricular end systolic volume (RVESV) $57 \mathrm{ml} / \mathrm{m}^{2}$ and right ventricular end diatolic volume (RVEDV) $157 \mathrm{ml} / \mathrm{m}^{2}$. 
Transcatheter pulmonary valve implantation was done under general anaesthesia. Heparin 100 $\mathrm{IU} / \mathrm{kg}$ was used when procedure was started. Broad spectrum antibiotic was given during cath. Venous check angiograms from both femoral veins was done to assess the size and morphology of the femoral veins for suitability of later introduction of large sheaths.

Angiogram in RAO 30 with $6 \mathrm{~F}$ pigtail through RFV at cran 15, 20ml at of $20 \mathrm{ml} / \mathrm{s}$ with 600 psi, Frame rate 15 frames/s done. Pressures of RV and the morphology of RV were noted.

Another injection with same catheter and projection in the main pulmonary artery was carried out. LAO 90 projection with pigtail in the MPA to see degree of stenosis and regurgitation, to estimate the shape and size of RVOT, narrowest pulmonary valve point, MPA size, take off of the LPA and to estimate length of the desired valve. The catheter is advanced to LPA to see the gradient across RVOT.

LOKUM Lunderquist extra-stiff guide wire $260 \mathrm{cms} 0.35$ wire" with $10 \mathrm{cms}$ PTFE coated straight tip (Cook Medical, IN, USA) was parked deep in the one of the branch of left pulmonary artery using $6 \mathrm{~F} \mathrm{MP}$ from LFV as RFV showed small caliber not suitable for large sheath. Via LFA 6F pigtail was placed in the LV to assess LVEDP. The catheter was then pulled back to aortic root for a later coronary angiogram during RVOT balloon inflation. Using the same (left) groin, another 6F pigtail is passed in the femoral vein and placed in the RV for check angiograms.

Balloon interrogation with $34 \mathrm{~mm}$ diameter/ $4 \mathrm{~cm}$ long, $75 \mathrm{~cm}$ shaft length Amplatzer TMASD Sizing Balloon Catheter (St. Jude Medical, MN, USA) was done over the Lunderquist wire and placed across the pulmonary valve deep in the main pulmonary artery. The balloon was then inflated using a contrast dilution of 1:5 and using a $\mathrm{T}$ tap at the end. Two syringes $50 \mathrm{ml}$ each were used with a two way tap at the balloon end. Enough contrast was injected into the balloon so that it completely occluded the RVOT and was fixed in the main pulmonary artery without any bobbing to and fro motion and was still at the end of inflation. A simultaneous injection was then given in the arterial root pigtail at $20 \mathrm{ml}$ and $20 \mathrm{ml} / \mathrm{s}$; 600 psi at LAO 90 projection. Patency of

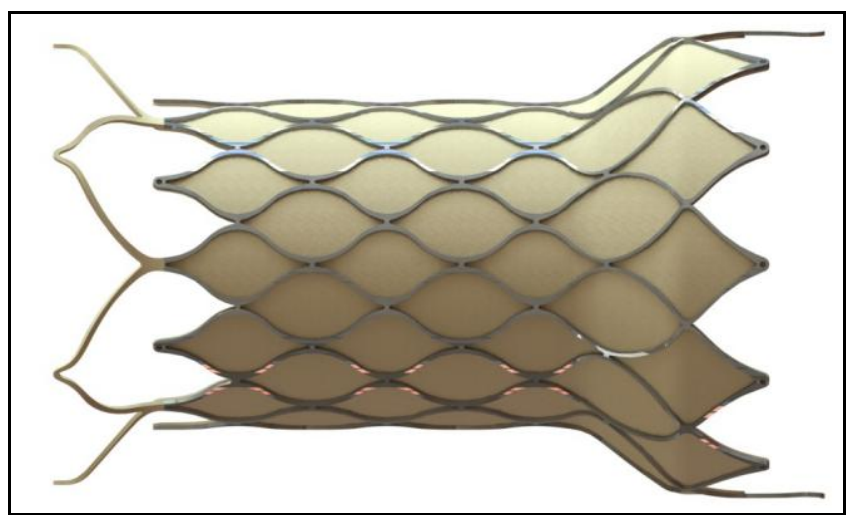

Figure-1: The Venus $p$-valve TM.

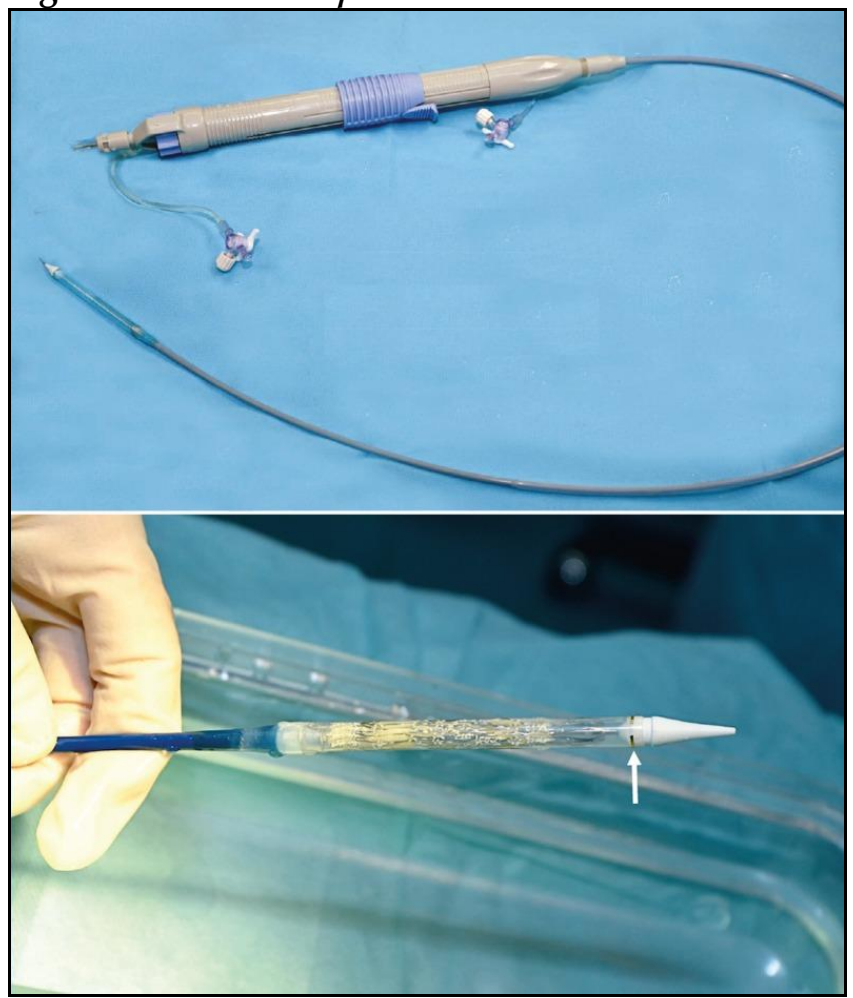

Figure-2: The Venus $p$-valve delivery system. note the handle with a knob for slow and controlled release of the valve and the distal capsule with a crimped and loaded valve inside. The arrow signs a distal radiopaque mark to be shifted to ICU.

the coronary arteries was checked and any compression of the arteries during balloon inflation of the RVOT was noted. In the same projection (LAO 90) a repeat angiogram was taken with 
$20 \mathrm{ml}$ contrast at $20 \mathrm{ml} / \mathrm{s}$ at $600 \mathrm{psi}$; using the $6 \mathrm{~F}$ pigtail placed in the RV cavity. Complete occlusion of the RVOT was achieved at maximum inflation of the sizing balloon (fig-3). No contrast was seen in the main pulmonary artery. The procedure was then repeated in the RAO 30, Cran 15

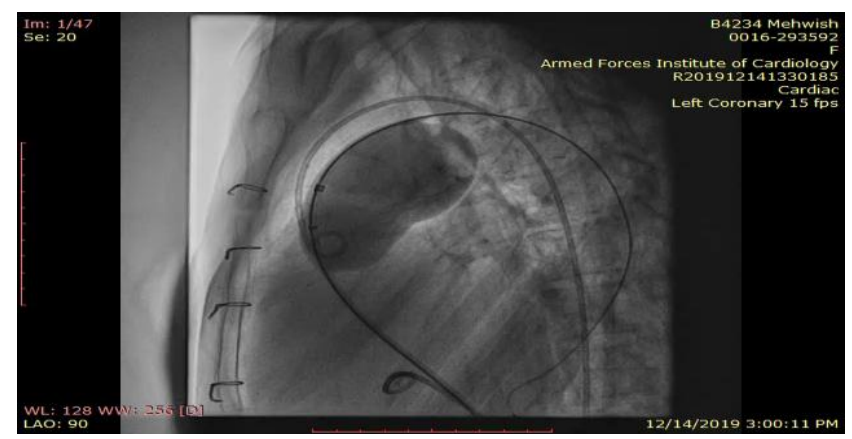

Figure-3: LAO 90 view showing complete occlusion of rvot with full inflation of balloon. This helped to estimate sizing of valve at the narrowest point.

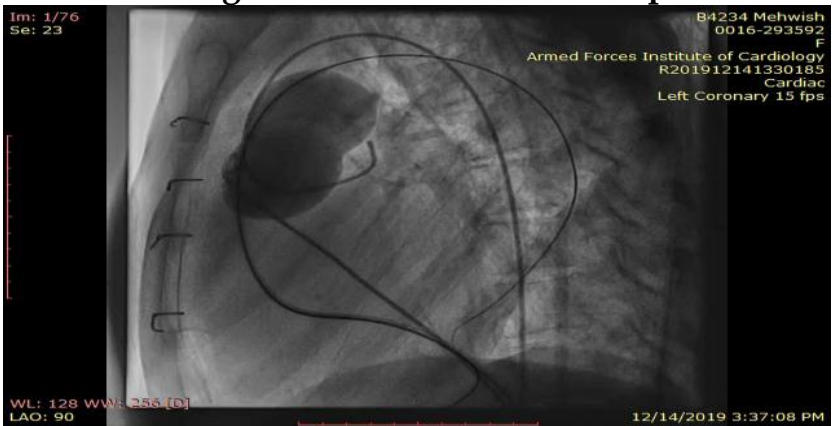

Figure-4: LAO 90 view showing LCA catheter away from full inlflated balloon.

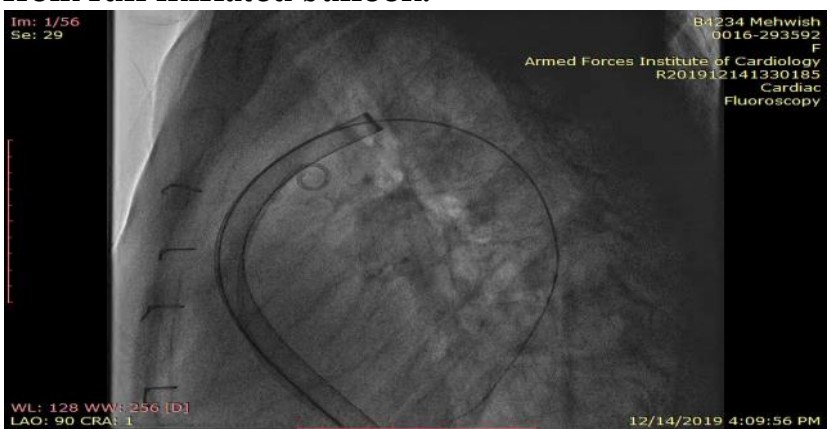

Figure-5: A 24 french sheath placed at the proximal LPA.

projection and check angiograms in the aortic root and in the RV cavity were repeated. Coronary compression in this new position was then ruled out during maximum compression of the sizing balloon using diluted contrast; injecting 80 $\mathrm{ml}$ with two $50 \mathrm{ml}$ syringes attached to the $\mathrm{T}$ tap (fig-4).
Predilating the left groin using $24 \mathrm{~F}$ Venus $p$ valve sheath dilator, repeated angiograms taken in LAO 90 and RAO 30 cranial 15, to keep distal end of sheath in to proximal LPA or along the anterior border of the trachea and so adjusted to avoid distal RPA origin. $6 \mathrm{~F}$ pigtail placed in the RV was then advanced into the MPA for later injections and pressure measurements. We selected Venus $p$-valve "shaft length $20 \mathrm{~mm}$ and with dia-

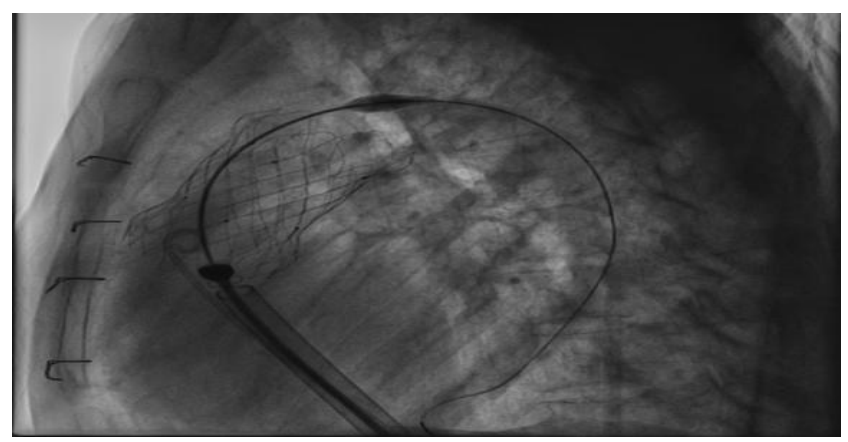

Figure-6: The self expanding valve is completely exposed from the sheath placed at optimal position.

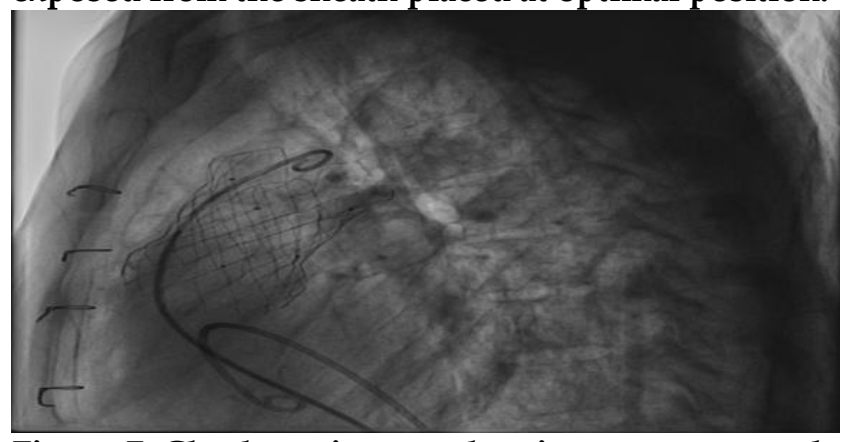

Figure-7: Check angiogram showing competent valve at pulmonary position.

meter of $34 \mathrm{~mm}^{\prime \prime}$ (fig-1). After rinsing with large amount of normal saline, the valve was manually crimped under cold normal saline onto a 22 French delivery system. Then, the whole system assembly was passed through a 24 French or larger Check-Flo® Performer Extra Large Introducer (Cook Medical, IN, USA), and placed at the proximal LPA (fig-5). Once in the optimal position, the valve was very slowly implanted by clockwise rotation of the releasing knob. Frequent pulmonary angiograms via pigtail catheter are performed to ensure a proper valve position until the valve was completely exposed (fig-6). After the valve deployment, the RV/ PA pressure and 
MPA angiography are repeated. The flair end of the valves was adjusted in the pain pulmonary artery and repeated check angiograms were taken in LAO 90 and RAO 25 making sure that the branch Pas remained unobstructed (fig-7). Repeated check angiograms were then taken and pressures recorded. Using a wire the pigtail in the PA was then gradually withdrawn. While withdrawing the $24 \mathrm{~F}$ sheath probably diaphragm of the sheath was damaged causing profuse bleeding, the sheath was immediately withdrawn with compression at the sheath site. Due to heavy bleeding, two units of blood were immediately replaced and she was resuscitated successfully to be shifted to ICU.

\section{DISCUSSION}

This was our initial experience of venus $p$ valve implantation in a patient in whom pericarial patch was used to reconstruct RVOT, with very favourable results. However case selection and technical expertise is key for the success.

\section{RECOMMENDATIONS}

- Injection Heparin 5000 IU every 6 hrly

- Injection Meropenem 500 mg 8 hrly

- Inj Amikin 500 mg BD

- Tab loprin $150 \mathrm{mg}$ OD indefinitly

- Tab Lowplat 75r mg OD for six months

- Tab paracetamol 2 tabs 8 hrly for 3 days

- Repeat ECHO after 24 hrs

- Ultrasound abdomen after 24 hrs for retroperitoneal bleed.

- Care of the access site.

- Follow up after 2 weeks, 3 months, 6 months, then 1 yearly

- MRI and fluoroscopy after 3 months to asses changes in the RV EDV/RV function and fracture of valve stents.

- Avoid Estrogen for contraception, use only progesterone to avoid thrombosis.

\section{CONCLUSION}

Venus $p$-valve is very effective and practical solution for Severe PR and failing RV post total correction. However we need to do more cases for more experience and data for future management of this category of patients.

\section{CONFLICT OF INTEREST}

This study has no conflict of interest to be declared by any author.

\section{REFERENCES}

1. McElhinney DB, Hellenbrand WE, Zahm EM. Short - and medium-term outcomes after transcatheter pulmonary valve placement in the expanded multicenter US melody valve trial. Circulation 2010; 122(5): 507-16.

2. Kenny D, Hijazi ZM, Kar S. Percutaneous implantation of the Edwards SAPIEN transcatheter heart valve for conduit failure in the pulmonary position: early phase 1 results from an international multicenter clinical trial. J Am Coll Cardiol 2011; 58(21): 2248-56.

3. Faza N, Kenny D, Kavinsky C, Amin Z, Heitschmidt M, Hijazi ZM. Single-center comparative outcomes of the Edwards SAPIEN and medtronic melody transcatheter heart valves in the pulmonary position. Catheter Cardiovasc Interv 2013; 82(1): e535-41.

4. Schievano S, Coats L, Migliavacca F, Pan W, Guan L, Ge J, et al. Variations in right ventricular outflow tract morphology following repair of congenital heart disease: implications for percutaneous pulmonary valve implantation. J Cardiovasc Magn Reson 2007; 9(1): 687-95.

5. Cao QL, Kenny D, Zhou D, Pan W, Guan L, Ge J, et al. Early clinical experience with a novel self-expanding percutaneous stent-valve in the native right ventricular outflow tract. Catheter Cardiovasc Interv 2014; 84(7): 1131-37.

6. Promphan W, Prachasilchai P, Siripornpitak S, Qureshi SA, Layangool T. Percutaneous pulmonary valve implantation with the venus p-valve: clinical experience and early results. Cardiol Young 2016; 26(4): 698-710.

7. Meadows JJ, Moore PM, Berman DP. Use and performance of the melody transcatheter pulmonary valve in native and postsurgical, non-conduit right ventricular outflow tracts. Circ Cardiovasc Interv 2014; 7: 374-80.

8. Boudjemline Y, Agnoletti G, Bonnet D, Sidi D, Bonhoeffer P. Percutaneous pulmonary valve replacement in a large right ventricular outflow tract: an experimental study. J Am Coll Cardiol 2004; 43: 1082-87.

9. Schievano S, Taylor AM, Capelli C. First-in-man implantation of a novel percutaneous valve: a new approach to medical device development. EuroIntervention 2010; 5(1): 745-50.

10. Fagan T, Kay J, Carroll J, Neubauer A. 3-D guidance of complex pulmonary artery stent placement using reconstructed rotational angiography with live overlay. Catheter Cardiovasc Interv 2012; 79(1): 414-21. 